\title{
Transverse emittance and current of multi-GeV trapped electrons in a plasma wakefield accelerator
}

\author{
N. Kirby, ${ }^{1}$ I. Blumenfeld, ${ }^{1}$ C. E. Clayton, ${ }^{2}$ F. J. Decker, ${ }^{1}$ M. J. Hogan, ${ }^{1}$ C. Huang, ${ }^{2}$ R. Ischebeck, ${ }^{1}$ R. H. Iverson, ${ }^{1}$ \\ C. Joshi, ${ }^{2}$ T. Katsouleas, ${ }^{3}$ W. Lu, ${ }^{2}$ K. A. Marsh, ${ }^{2}$ S. F. Martins, ${ }^{4}$ W. B. Mori, ${ }^{2}$ P. Muggli, ${ }^{3}$ E. Oz, ${ }^{3}$ R. H. Siemann, ${ }^{1}$ \\ D. R. Walz, ${ }^{1}$ and M. Zhou ${ }^{2}$ \\ ${ }^{1}$ SLAC, Menlo Park, California 94025, USA \\ ${ }^{2}$ University of California, Los Angeles, California 90095, USA \\ ${ }^{3}$ University of Southern California, Los Angeles, California 90089, USA \\ ${ }^{4}$ GoLP/IPFN, Instituto Superior Técnico, Portugal \\ (Received 17 December 2008; published 14 May 2009)
}

\begin{abstract}
Multi-GeV trapped electron bunches in a plasma wakefield accelerator (PWFA) are observed with normalized transverse emittance divided by peak current, $\epsilon_{N, x} / I_{t}$, below the level of $0.2 \mu \mathrm{m} / \mathrm{kA}$. A theoretical model of the trapped electron emittance, developed here, indicates that emittance scales inversely with the square root of the plasma density in the nonlinear "bubble" regime of the PWFA. This model and simulations indicate that the observed values of $\epsilon_{N, x} / I_{t}$ result from multi-GeV trapped electron bunches with emittances of a few $\mu \mathrm{m}$ and multi-kA peak currents.
\end{abstract}

Low emittance electron bunches have applications that include next generation light sources and particle accelerators [1]. At present, thermionic and photoemission based electron sources are widely used [2]; however, plasma based electron sources are an active topic of research due to their potential to produce high current and low emittance electron bunches [3-7]. Recently, it was shown that plasma wakefield accelerators (PWFAs) [8-10], operated in the nonlinear bubble regime, can trap electrons that are released by ionization inside the plasma wake [11] and accelerate them to high energies. In this paper, a combination of measurements, theory, and simulations show that multi-GeV trapped electron bunches from a PWFA achieve emittances of a few $\mu \mathrm{m}$ and multi-kA peak currents. The theory, presented here, derives a scale for the minimum achievable emittance for these electrons, which is also relevant for trapped electrons from a laser wakefield accelerator (LWFA) [12] in the bubble regime.

In this experiment, an ultrarelativistic electron drive bunch field ionizes neutral vapor, creating a plasma $[13,14]$. The density of the drive bunch exceeds that of the plasma, so the bunch expels all the plasma electrons from the region around it. The ions then pull the plasma electrons back to the axis of bunch propagation with a time scale set by the inverse of the plasma frequency, $\omega_{p}=$ $\sqrt{n_{p} e^{2} /\left(m \epsilon_{0}\right)}$, where $n_{p}$ is the plasma density. This produces a bubble containing a region of uniformly charged ions behind the drive bunch (see Fig. 1), which is characteristic of the bubble regime.

Trapping then occurs in the resultant plasma wake due to the presence of two gas species in the vapor: in this experiment, helium and lithium, with ionization energies of 24.6 and $5.4 \mathrm{eV}$, respectively. A heat-pipe oven is the source for the neutral vapor [15]. Over the central heated region of this oven there is pure lithium vapor with a density of $2.7 \times 10^{23} \mathrm{~m}^{-3}$ and a FWHM length of $85 \mathrm{~cm}$, but in the cool regions on either side of the heated region there is pure helium. In between, there is a transition region where both species are present (see Fig. 3). Ionization of lithium atoms occurs first from the bunch electric field, releasing the electrons that support the plasma wake. As the fields of the bunch and the wake increase, the helium atoms become ionized inside the wake and are a source of the observed trapped electrons [11], as described below.

Trapping of electrons that are released inside a plasma wake is addressed with a constant of motion, $\gamma m c^{2}-$ $c p_{z}-e\left(\Phi-c A_{z}\right)$, where $\Phi, A, \gamma$, and $p$ denote the scalar potential, the vector potential, the electron's Lorentz factor,

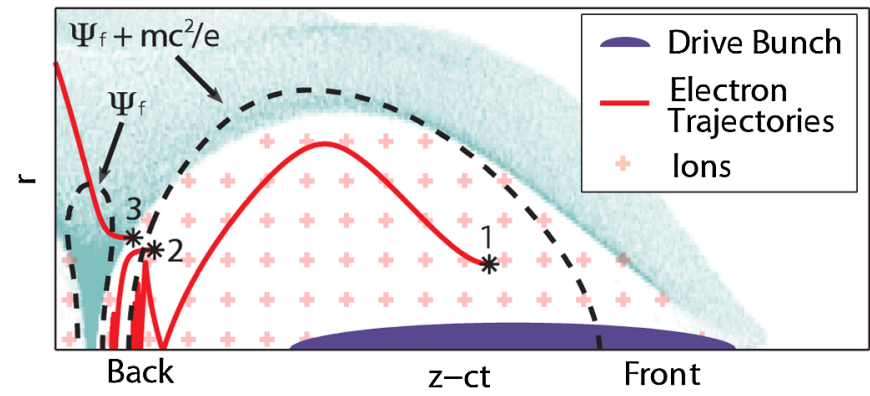

FIG. 1. (Color) An illustration of electron trapping, calculated with OSIRIS [22]. A few example helium electron trajectories overlay an image of lithium electron density (teal), where the asterisks display the ionization location, and the dotted lines represent $\Psi$ contours. The electrons that escape their atoms within the $\Psi_{f}+m c^{2} / e$ contour ( 1 and 2 ) become trapped, while electron 3 slips out of the wake. 
and momentum, respectively [16]. Let $\Psi$ denote $\Phi-c A_{z}$. Calculations use cylindrical coordinates, $(r, \phi, z)$, where $z$ denotes the direction of drive bunch propagation, assuming cylindrical symmetry, $\partial_{\phi}=0$, and zero azimuthal current, $J_{\phi}=0$. Since ionization releases electrons near rest, the constant of motion is $m c^{2}-e \Psi_{i}$ and $\Psi_{i}-\Psi<m c^{2} / e$ (from $\gamma m c^{2}-c p_{z}>0$ ). Partial derivatives relate $\Psi$ to the electric and magnetic fields: $\partial_{r} \Psi=-\left(E_{r}-c B_{\phi}\right)$ and $\partial_{z} \Psi=-E_{z}$. The accelerating field is related to the radial current, $\partial_{r} E_{z}=\mu_{0} c J_{r}$ [17], and inside the bubble $E_{r}-$ $c B_{\phi}=n_{p} e r /\left(2 \epsilon_{0}\right)$ [18]. The quantity $\Psi$ reaches a maximum, $\Psi_{m}$, on axis at the center of the bubble, where its partial derivatives are zero and the radius of the bubble, $R$, reaches a maximum, $R_{m}$. Let $\Psi_{f}$ denote $\Psi$ on axis at the back of the bubble, where fields change from accelerating and focusing to either decelerating or defocusing. An electron that satisfies $\Psi_{i}>m c^{2} / e+\Psi_{f}$ cannot slip out of the back of the bubble, as Fig. 1 illustrates; thus, it becomes trapped in the wake.

This trapping condition between $\Psi_{i}$ and $\Psi_{f}$ is now connected to $R_{m}$ and to the drive bunch peak current, $I_{d}$. An integration of $\partial_{r} \Psi$ gives $\Psi(r=0)=\int_{0}^{\infty} d r\left(E_{r}-\right.$ $\left.c B_{\phi}\right)$. The difference between $\Psi_{m}$ and $\Psi_{f}$ is dominated by the difference in their corresponding integrals over the interior of the bubble. Considering only this part of the integral results in $\Psi(r=0)=\int_{0}^{R} d r\left(E_{r}-c B_{\phi}\right)$, which implies $\Psi_{f}=0$ and $\Psi_{m}=n_{p} e R_{m}^{2} /\left(4 \epsilon_{0}\right)$. For trapping to be possible $\Psi_{m}-\Psi_{f}>m c^{2} / e$, which implies that $R_{m}>$ $2 / k_{p}$. This maximum radius can then be connected to the drive bunch as $R_{m} k_{p} / 2 \approx \sqrt{2 I_{d} / I_{A}}$ [19], where $I_{A} \approx$ $17 \mathrm{kA}$ is the Alfvén current. Note, accounting for the part of the integration of $\partial_{r} \Psi$ that is outside the bubble allows electron trapping to occur with $I_{d}$ slightly below $I_{A} / 2$. Thus, $I_{A} / 2$ only sets the scale for $I_{d}$ required to induce electron trapping.

The trapping requirements on the plasma wake and drive bunch, just developed, lead to a relationship between the trapped electron emittance and $c / \omega_{p}=1 / k_{p}$. Each plasma electron has a radial position and zero momentum when it escapes its atom. The focusing fields then rotate the electrons in the $x-p_{x}\left(\right.$ or $\left.y-p_{y}\right)$ plane, where $x=$ $r \cos \phi$, and $\theta$ denotes the angle in this plane. As the drive bunch propagates through the plasma and the trapped electrons rotate in this plane, the wake collects additional electrons at $\theta=0$, resulting in a uniform distribution in $\theta$ and a finite emittance. The focusing forces are linear in $x$, so after the phase space is filled there is no additional emittance growth. Thus, the first phase space rotation determines the transverse normalized emittance, expressed in Eq. (1):

$$
\epsilon_{N, x}=\frac{1}{m c} \sqrt{\left\langle x^{2}\right\rangle\left\langle p_{x}^{2}\right\rangle-\left\langle x p_{x}\right\rangle^{2}}
$$

As the trapped electrons accelerate in the wake, the focus- ing force applied to them changes from one given by just $E_{r}$ to $E_{r}-c B_{\phi}$. The exact trajectory of the electrons in the $x-p_{x}$ plane depends on the specifics of this transition. An appropriate assumption for $E_{z}$ in the back of the bubble is $\partial_{z} E_{z}=n e /\left(2 \epsilon_{0}\right) \quad$ [20]. Under this assumption $E_{r}=$ $n_{p} e r /\left(4 \epsilon_{0}\right)$, which is only a factor of 2 smaller than $E_{r}-$ $c B_{\phi}$. Therefore, either $E_{r}$ or $E_{r}-c B_{\phi}$ can be used as the focusing field to set the scale for the emittance. Electrons only become moderately relativistic during the first rotation, so a nonrelativistic harmonic oscillator approximates their motion. The quantity $E_{r}$ then sets the $x$ focusing force as $-n_{p} e^{2} x /\left(4 \epsilon_{0}\right)$. Let the trapped electrons have an initial mean square size in $x$ of $\left\langle x_{0}^{2}\right\rangle$ and zero transverse momentum. Since $x=x_{0} \cos \theta$, then $\left\langle x^{2}\right\rangle=\left\langle x_{0}^{2}\right\rangle / 2$. The size in $x$ determines the size in $p_{x},\left\langle p_{x}^{2}\right\rangle=m^{2} c^{2} k_{p}^{2}\left\langle x^{2}\right\rangle / 4$, and the uniform distribution of $\theta$ means $\left\langle x p_{x}\right\rangle=0$. Thus, $\epsilon_{N, x}=$ $k_{p}\left\langle x_{0}^{2}\right\rangle / 4$.

The location of ionization within the wake determines $\left\langle x_{0}^{2}\right\rangle$, with ionization occurring in regions of high electric field. The magnitude of $E_{z}$ peaks at the front and back of the bubble, and $E_{r}$ peaks at the front, near the drive bunch. Electron trapping only occurs if $R$ exceeds $2 / k_{p}$, so the drive bunch expels the trapped electrons that are released by ionization near it to similar radial distances. This sets a minimum scale of $\left\langle x_{0}^{2}\right\rangle \approx 1 / k_{p}^{2}$ in the front.

Analysis of $\left\langle x_{0}^{2}\right\rangle$ for the electrons that escape from their atoms in the back of the bubble is more detailed and requires an understanding of the field ionization process. The quantity $\Psi_{i}$ controls whether an electron becomes trapped and its final $z-c t$ location in the bubble. Thus, any longitudinal location in a trapped electron bunch is composed of electrons that escape their atoms along the same $\Psi$ contour. Recall Fig. 1, electrons released along the $\left(\Psi_{f}+m c^{2} / e\right)$ contour eventually gather at the $\Psi_{f}$ contour on axis, despite possessing different initial $z-c t$ locations. Therefore, $\left\langle x_{0}^{2}\right\rangle$ for the electrons released in the back of the bubble results from the transverse size of the ionization rate along the $\Psi$ contours.

The initial transverse size for the trapped electrons on a $\Psi$ contour also has a minimum scale, which is imposed by the position of their release. For electrons to escape their atoms in the back of the bubble, $E_{z}$ must at least be on the order of that in the front $\left(m c \omega_{p} / e\right)$. Let $z-c t=0$ denote the middle of the bubble, where $E_{z}=0$. Consider a $\Psi$ contour at the back of the bubble with $E_{z}=-m c \omega_{p} / e$ on axis. This contour roughly corresponds to the earliest position in the back of the bubble where ionization occurs. Assuming $\partial_{z} E_{z}=n e /\left(2 \epsilon_{0}\right)$, this occurs at $z-c t=$ $-2 / k_{p}$. Figure 2(a) shows the magnitude of the electric field, $|E|$, as a function of $r$ along this $\Psi$ contour (denoted by $\left.E_{0}\right)$. As illustrated by the $\left(\Psi_{f}+m c^{2} / e\right)$ contour in Fig. 1, the contours reach their maximum radius in the middle of the bubble. This maximum radial extent sets the scale for the initial transverse size. The $\Psi$ contour corre- 

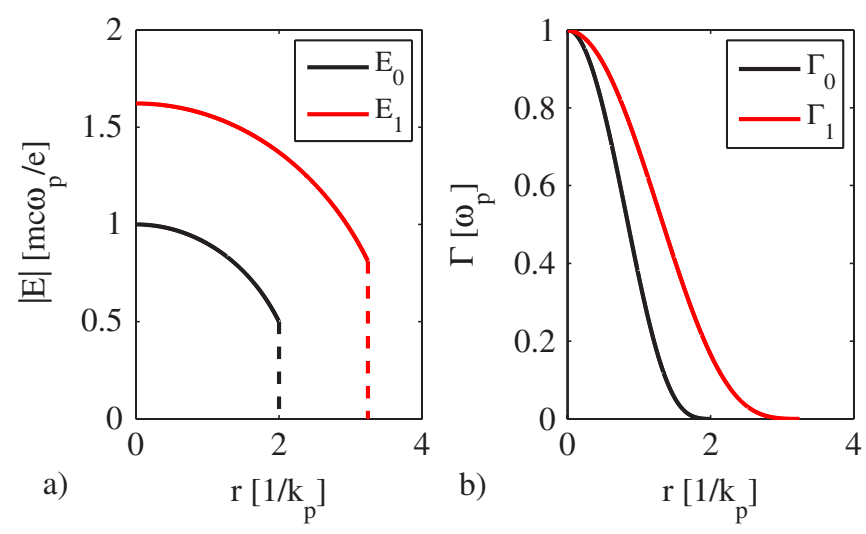

FIG. 2. (Color) (a) The magnitude of the electric field along a $\Psi$ contour with $z-c t=-2 / k_{p}$ on axis, $E_{0}$; and with $z-c t=$ $-3.244 / k_{p}$ on axis, $E_{1}$. The radial extent of a contour increases as it moves farther back in the bubble. (b) The ionization rate of helium for $E_{0}$ at $n_{p}=8.0 \times 10^{23} \mathrm{~m}^{-3}, \Gamma_{0}$, and for $E_{1}$ at $n_{p}=$ $2.7 \times 10^{23} \mathrm{~m}^{-3}, \Gamma_{1}$.

sponding to $E_{0}$ has a maximum radial extent of $2 / k_{p}$. At positions farther back in the bubble this radial extent increases, as displayed in Fig. 2(a), so the initial transverse size created by $E_{0}$ is representative of the minimum achievable.

The field ionization formula [13] is now applied to $|E|$ along the $\Psi$ contour to derive $\left\langle x_{0}^{2}\right\rangle$ in the back of the bubble. Since the time scale for the duration of the bubble is $1 / \omega_{p}$, the ionization rate must be on the order of $\omega_{p}$ to cause ionization inside the bubble. At a plasma density of $2.7 \times 10^{23} \mathrm{~m}^{-3}$ the ionization rate of helium created by $E_{0}$ is too small for electrons to be released along its corresponding $\Psi$ contour. For this density the ionization rate equals $\omega_{p}$ at $z-c t=-3.244 / k_{p}$. Figure 2(a) shows $|E|$ along this $\Psi$ contour, $E_{1}$; and Fig. 2(b) shows the corresponding ionization rate, $\Gamma_{1}$, as a function of $r$. The radial extent of this rate corresponds to $\left\langle x_{0}^{2}\right\rangle=0.92 / k_{p}^{2}$. Similarly, the ionization rate from $E_{0}$ for helium equals $\omega_{p}$ at a density of $8.0 \times 10^{23} \mathrm{~m}^{-3}$. Figure 2(b) shows the ionization rate corresponding to $E_{0}$ at this plasma density, $\Gamma_{0}$, as a function of $r$. The radial extent of this rate corresponds to $\left\langle x_{0}^{2}\right\rangle=0.36 / k_{p}^{2}$.

Both ionization locations, front and back, therefore have a minimum initial size of order $1 / k_{p}$. As the trapped electron bunches are composed of electrons from both ionization locations, it is appropriate to characterize the minimum achievable emittance with $\left\langle x_{0}^{2}\right\rangle \approx 1 / k_{p}^{2}$. This yields a minimum value of $\epsilon_{N, x}=1 /\left(4 k_{p}\right)$, which is proportional to $n_{p}^{-1 / 2}$. The previous paragraph indicates that an initial transverse size can occur slightly below $1 / k_{p}$, so $1 /\left(4 k_{p}\right)$ only sets the scale for the minimum achievable emittance. Since electrons in a LWFA are expelled to radial distances significant compared to $1 / k_{p}$ before becoming trapped, similar arguments and scales are applicable for the emittance of these electron bunches.

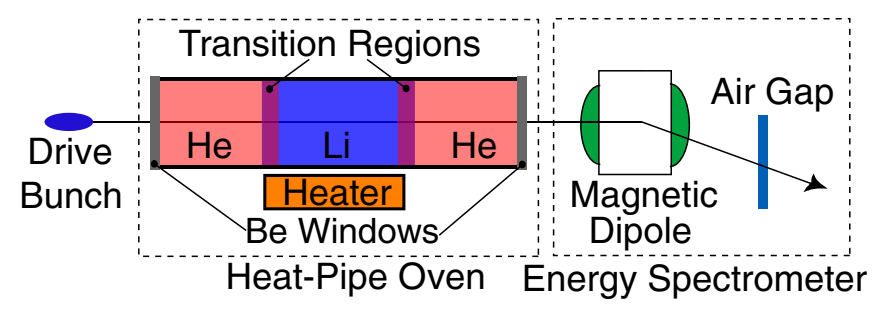

FIG. 3. (Color) The experimental layout of the heat-pipe oven and the energy spectrometer.

The trapped electrons are characterized with PWFA experiments conducted at the Final Focus Test Beam (FFTB) facility, located at the end of the Stanford Linear Accelerator Center linac. The $42 \mathrm{GeV}$ electron drive bunches have $1.8 \times 10^{10}$ electrons, normalized emittances of $\epsilon_{N, x}=60 \mu \mathrm{m}$ and $\epsilon_{N, y}=7 \mu \mathrm{m}$, and longitudinal bunch lengths of tens of $\mu \mathrm{m}\left(I_{d} \approx I_{A}\right)$. A measurement of the drive bunch energy spectrum at the beginning of the FFTB beam line uniquely determines the drive bunch's longitudinal phase space [9]. The bunch then passes through a $1 \mu \mathrm{m}$ thick Ti foil. A pyroelectric detector collects coherent transition radiation from this foil for use as a complementary bunch length measurement. Next, the drive bunch, with a transverse size of $10 \mu \mathrm{m}$, enters the heat-pipe oven (see Fig. 3). Beryllium windows of thickness 50 and $75 \mu \mathrm{m}$ are located up and downstream of the heat-pipe oven, respectively, and form boundaries between the helium buffer gas and the beam line vacuum. Next, the drive bunch and the trapped electrons pass through an energy spectrometer, which consists of a magnetic dipole and an air gap. An optical system focuses Cherenkov radiation from the air gap onto a cooled charge-coupled device camera [8]. Figure 4(a) shows a sample image from the energy spectrometer air gap.

In addition to the signature for energy loss on the drive bunch, when $I_{d}$ approaches $I_{A} / 2$, narrow electron streaks

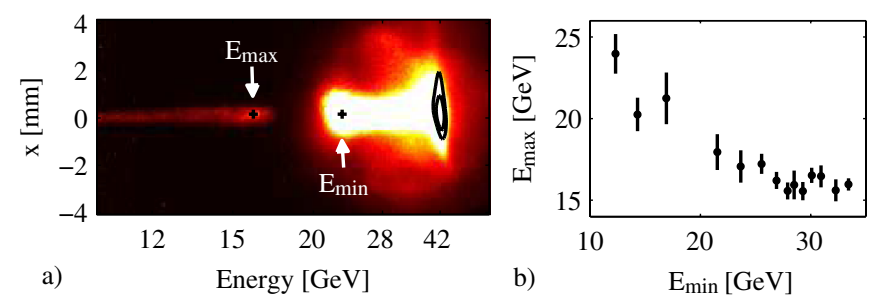

FIG. 4. (Color) (a) A saturated image from the energy spectrometer, where the black contour lines show the drive bunch spot size in the absence of plasma acceleration. Trapped electrons appear as a streak with an rms $x$ size that is much smaller than that of the drive bunch. The black plus signs denote the maximum energy of the electron streak, $E_{\max }$, and the minimum energy of the drive bunch, $E_{\min }$, after accounting for the natural spot size of the electron bunches [26]. (b) The quantity $E_{\max }$ versus $E_{\min }$, where the difference between the streak and drive bunch $x$ rms size allows for a measurement of $E_{\max }$, even when it extends above $E_{\min }$. 
appear in the spectrometer images. Since the streaks show up with energies lower than the initial drive bunch energy, they are the result of either the wake accelerating plasma electrons from $0 \mathrm{GeV}$ or decelerating drive bunch electrons from $42 \mathrm{GeV}$. A variation of the drive bunch longitudinal length changes the magnitude of $E_{z}$ in the wake. Figure 4(b) shows that, as stronger plasma wakes decrease the minimum energies of the drive bunches, the maximum energies of the electron streaks increase, so the streaks must be in the accelerating part of the wakes. Thus, the streaks are trapped plasma electrons.

An examination of the propagation for the trapped electrons from the heat-pipe oven to the energy spectrometer leads to a measurement of $\epsilon_{N, x} / I_{t}$, where $I_{t}$ is the trapped electron peak current. Plasma electrons first become trapped at the upstream helium-lithium transition region. The drive bunch's wake then accelerates these trapped electrons through the $85 \mathrm{~cm}$ of lithium. Next, the electrons pass into the downstream pure helium region of the oven. Simulations, presented later, show that the trapped electrons have fields large enough to ionize helium. After the drive bunch stops ionizing helium it diverges freely; however, the trapped electrons create an ion column that confines them transversely as they propagate through the helium buffer gas [21]. These trapped electrons diverge freely either before or when they reach the downstream beryllium window. Thus, the rms $x$ size on the spectrometer, $\sigma$ [from images similar to Fig. 4(a)] divided by the length from the beryllium window to this spectrometer, $L=193 \mathrm{~cm}$, is an upper limit measurement of the trapped electron bunch angular divergence.

Once the electric field of the trapped electrons cannot ionize helium, they diverge freely; this sets a maximum transverse size for the trapped electrons as they begin to diverge. The peak electric field of a trapped electron bunch with $\sigma_{x}=\sigma_{y}$ is proportional to $I_{t} / \sigma_{x}$, where $\sigma_{x}$ and $\sigma_{y}$ represent the bunch's $x$ and $y \mathrm{rms}$ size, respectively. For a given longitudinal bunch length, the maximum transverse size capable of ionizing helium, $\sigma_{m}$, is proportional to the peak current, $\sigma_{m}=\alpha I_{t}$; here, $\alpha$ is the proportionality constant when the ionization probability [13] is equal to 0.5 at the peak field position of a Gaussian bunch. This constant is only weakly dependent on the bunch length: for $\sigma_{z}=0.5,1.9$, and $5 \mu \mathrm{m}, \alpha=2.39,2.83$, and $3.14 \times$ $10^{-10} \mathrm{~m} / \mathrm{A}$, respectively [21]. The cores of the trapped electron bunches from the simulations of the experiment, presented later, are well represented by Gaussian bunches with $\sigma_{z}$ in the range of 1.1 to $2.7 \mu \mathrm{m}$. Since $\sigma_{z}=1.9 \mu \mathrm{m}$ is in the middle of this range, the corresponding value of $\alpha$ is most appropriate. Thus, the maximum transverse size the trapped bunches are at the point they start to diverge freely is $\sigma_{m}=\alpha I_{t}$.

Until the bunch begins to diverge freely, the $\left\langle x p_{x}\right\rangle$ term, in Eq. (1), is small. Let the coordinate $\dot{x}=p_{x} / p_{z}$. Equation (1) then reduces to $\epsilon_{N, x}<\gamma \sigma_{x} \sigma_{\dot{x}}$, where $\sigma_{\dot{x}}$
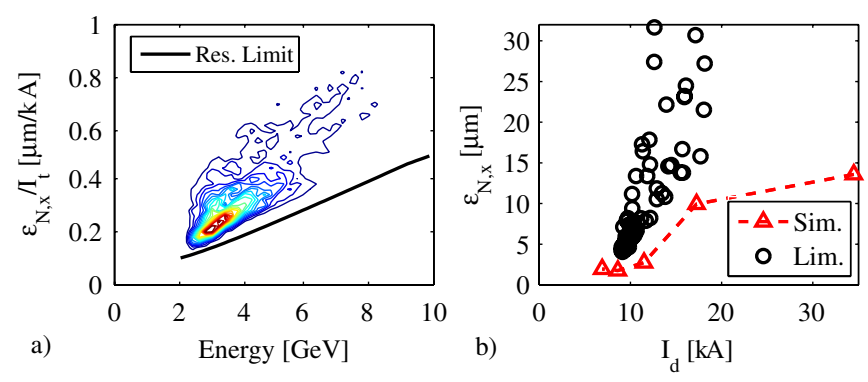

FIG. 5. (Color) (a) A contour plot of the measured upper limit for $\epsilon_{N, x} / I_{t}$ vs energy, obtained from Eq. (2), where the black line represents the system resolution. (b) The upper limit of $\epsilon_{N, x}$ for the 65 events from the experiment (Lim.) is consistent with the values from simulations ( $\mathrm{Sim}$.).

represents the $\dot{x}$ rms size of the bunch. The substitution of $\sigma_{m}$ and $\sigma / L$ into this upper limit for $\epsilon_{N, x}$ results in an upper limit measurement of $\epsilon_{N, x} / I_{t}$,

$$
\frac{\epsilon_{N, x}}{I_{t}}<\frac{\gamma \alpha \sigma}{L}
$$

For each experimental event, the trapped bunch is divided into energy bins. A measurement of $\sigma$ for each bin results in an upper limit of $\epsilon_{N, x} / I_{t}$ for each bin. The bins from a set of events form the contour plot in Fig. 5(a).

Three-dimensional particle in cell simulations of the experiment, using the code OSIRIS [22], provide support to the trapped electron $\epsilon_{N, x}$ calculations. These simulations have a lithium density of $2.7 \times 10^{23} \mathrm{~m}^{-3}$ and a relative helium density of $3 \%$. The presence of this relatively small helium density does not produce a large effect on the fields of the wake so it does not effect the rotation of trapped electrons in the $x-p_{x}$ plane or their transverse emittance. As in the experiment, the helium only serves as a source for the trapped electrons. The simulation grid size in $x, y$, and $z$ is $0.5 \mu \mathrm{m}$ with either 2 or 4 particles per cell (second order interpolation [23]). These simulations are for Gaussian drive bunches with $1.8 \times 10^{10}$ electrons, matched transverse sizes of $\sigma_{x, y}=1.74 \mu \mathrm{m}$, and $I_{d}=34.5,17.2,11.5$, 8.62 , and $6.90 \mathrm{kA}$; the quantity of trapped electrons dramatically reduces for $I_{d}<6.90 \mathrm{kA}$. Trapped electrons are not of a single energy, so $\epsilon_{N, x}$ is calculated in energy bins. In these simulations, the values of $\epsilon_{N, x}$ for the trapped electrons in descending $I_{d}$ order are 13.6 $\pm 2.0,9.94 \pm$ $0.33,2.74 \pm 0.61, \quad 1.76 \pm 0.43$, and $1.95 \pm 0.69 \mu \mathrm{m}$, shown in Fig. 5(b). These values correspond to the mean and rms of $\epsilon_{N, x}$ from a charge weighting of the energy bins. The relative small size of the rms spreads confirm that $\epsilon_{N, x}$ is weakly dependent on the energy. These simulations indicate that trapped electrons can achieve $\epsilon_{N, x}$ at a fraction of $1 / k_{p}$, as predicted by the model $\left(1 / k_{p} \approx 10 \mu \mathrm{m}\right)$. Additional simulations at plasma densities significantly greater and lower than $2.7 \times 10^{23} \mathrm{~m}^{-3}$ confirm the $1 / k_{p}$ scaling of the trapped electron emittance [24]. 
Once enough electrons become trapped, they load the accelerating field and cease additional trapping. The OSIRIS simulations run until the trapped electrons load the wake and turn off further trapping, resulting in a maximum achievable trapped electron peak current, $I_{t, m}$. For $I_{d}=$ $34.5,17.2,11.5,8.62$, and $6.90 \mathrm{kA}, I_{t, m}=125,80.3,42.2$, 17.3 , and $2.60 \mathrm{kA}$, respectively. Even at the lowest energies used in the measurement of $\epsilon_{N, x} / I_{t}$, the matched spot sizes for these simulated trapped electron bunches divided by their current are below $\alpha$, indicating they can ionize helium.

From the simulated relationship between $I_{d}$ and $I_{t, m}$, a likely $I_{t, m}$ is determined for each experimental event; this value combined with the upper limit for $\epsilon_{N, x} / I_{t}$ results in an upper limit of $\epsilon_{N, x}$ for each event. The trapped electron bunches appear with rms $x$ sizes near the system resolution [25]. Thus, as $\gamma$ increases so does the upper limit of $\epsilon_{N, x} / I_{t}$; this is a consequence of the system resolution instead of a property of the trapped electrons. Both the model and simulations indicate $\epsilon_{N, x}$ is not very dependent on energy, so the upper limit of $\epsilon_{N, x} / I_{t}$ for each event comes from an average over a low energy interval of 2.3 to $3.0 \mathrm{GeV}$, where there is the best resolution. Figure 5(b) shows the determined upper limits from the experiment. The minimum value achieved for the upper limit of $\epsilon_{N, x}$ is $4 \mu \mathrm{m}$, which is close to $1 /\left(4 k_{p}\right)(2.5 \mu \mathrm{m})$ and smaller than the drive bunch emittance.

Similarly, the theoretical model shows there is a minimum scale for $\epsilon_{N, x}$; a combination of this scale with the measured upper limit for $\epsilon_{N, x} / I_{t}$ results in a lower limit for $I_{t}$. Since simulations achieve emittance below $1 /\left(4 k_{p}\right)$, then the minimum emittance in the simulations, $1.76 \mu \mathrm{m}$, is used for the minimum emittance scale. The experimental shot with a $4 \mu \mathrm{m}$ upper limit for $\epsilon_{N, x}$ has a measured value of $\epsilon_{N, x} / I_{t}<0.19 \mu \mathrm{m} / \mathrm{kA}$, which suggests that $I_{t}$ is greater than $9.2 \mathrm{kA}$.

Systematic errors dominate the measurement uncertainties. These errors include the system resolution and the neglect of $\left\langle x p_{x}\right\rangle$. The net result of these errors is a measurement that is systematically larger than the actual $\epsilon_{N, x} / I_{t}$, which causes the determined values for $\epsilon_{N, x}$ and $I_{t}$ to be systematically larger and smaller, respectively, than the actual values.

A theoretical model, presented here, indicates that the scale of the achievable trapped electron transverse normalized emittance is a fraction of the plasma length scale, $1 / k_{p} \propto n_{p}^{-1 / 2}$, in the nonlinear bubble regime of the PWFA. It is not limited by the emittance of the drive bunch. The model is consistent with both simulations and experimental measurements. A combination of these measurements and simulations indicates that multi-GeV trapped electron bunches have simultaneously achieved
$\epsilon_{N, x}$ better than $4 \mu \mathrm{m}$ with a peak current greater than $9.2 \mathrm{kA}$.

\section{ACKNOWLEDGMENTS}

The authors would like to thank Melissa Berry and Professor Alexander Chao. The Dawson cluster (UCLA) produced the OSIRIS simulations. This work was supported by Department of Energy Contracts No. DE-AC0276SF00515, No. DE-FG02-93ER40745, No. DE-FG0392ER40727, No. DE-FG52-06NA26195, No. DE-FC0207ER41500, No. DE-FG02-03ER54721, No. DE-FG0292ER40727, National Science Foundation Grant No. NSF-Phy-0321345, and by FCT (Portugal).

[1] M. J. Van Der Weil, in ICFA Workshop on the Physics of High Brightness Beams (World Scientific, Los Angeles, CA, 1999), p. 3.

[2] C. Hernandez-Garcia et al., Phys. Today 61, No. 2, 44 (2008).

[3] C. B. Shroeder et al., in Proceedings of FEL (BESSY, Berlin, 2006), p. 455.

[4] S. Fritzler et al., Phys. Rev. Lett. 92, 165006 (2004).

[5] S.-Y. Chen et al., Phys. Plasmas 6, 4739 (1999).

[6] C. G.R. Geddes et al., Nature (London) 431, 538 (2004).

[7] A. W. Chao et al., Technical Report No. SLAC-PUB-9189, Stanford Linear Accelerator Center, 2002.

[8] I. Blumenfeld et al., Nature (London) 445, 741 (2007).

[9] M. J. Hogan et al., Phys. Rev. Lett. 95, 054802 (2005).

[10] P. Muggli et al., Phys. Rev. Lett. 93, 014802 (2004).

[11] E. Oz et al., Phys. Rev. Lett. 98, 084801 (2007).

[12] W. Leemans and E. Esarey, Phys. Today 62, No. 3, 44 (2009), and references therein.

[13] D. L. Bruhwiler et al., Phys. Plasmas 10, 2022 (2003).

[14] C.L. O'Connell et al., Phys. Rev. ST Accel. Beams 9, 101301 (2006).

[15] P. Muggli et al., IEEE Trans. Plasma Sci. 27, 791 (1999).

[16] P. Mora and T. M. Antonsen, Jr., Phys. Plasmas 4, 217 (1997).

[17] R. J. Briggs, Phys. Rev. Lett. 54, 2588 (1985).

[18] J. B. Rosenzweig et al., Phys. Rev. A 44, R6189 (1991).

[19] W. Lu et al., Phys. Plasmas 13, 056709 (2006).

[20] W. Lu et al., Phys. Rev. Lett. 96, 165002 (2006).

[21] N. Kirby et al., Ref. [26], p. 591.

[22] R. A. Fonseca et al., in Proceedings of the International Conference on Computational Science-Part III (SpringerVerlag, London, 2002), p. 342.

[23] T. Esirkepov, Comput. Phys. Commun. 135, 144 (2001).

[24] N. Kirby, Ph.D. thesis, Stanford University, 2009.

[25] N. Kirby et al., in Proceedings of the Particle Accelerator Conference, Albuquerque, New Mexico, 2007 (IEEE, Albuquerque, New Mexico, 2007), p. 4183.

[26] I. Blumenfeld et al., in Advanced Accelerator Concepts (AIP, Santa Cruz, CA, 2008), p. 569. 